\title{
Semicarbazide-Sensitive Amine Oxidase (SSAO) Gene Expression in Alloxan-induced Diabetes in Mice
}

\author{
Jenny L. E. Nordquist, Camilla Göktürk, and Lars Oreland \\ Department of Neuroscience, Unit of Pharmacology, Uppsala University, Uppsala, Sweden \\ Accepted October 1, 2002
}

\begin{abstract}
Background: Plasma activity of semicarbazide-sensitive amine oxidase (SSAO) has been reported to be significantly higher in diabetic patients compared to healthy controls. Due to the production of highly angiotoxic substances in SSAO-catalyzed reactions, it has been speculated that this could be a cause for the vascular complications frequently associated with diabetes. Little is known about how the enzyme activity is regulated, and why it is high in these patients. In the present study, we assessed the possibility of transcriptional regulation by analyzing SSAO activity and SSAO-mRNA levels in mice with alloxan-induced diabetes. Materials and Methods: Diabetes was induced in NMRI mice by a single intravenous injection of alloxan. The enzyme activity was analyzed by a radiometric assay using ${ }^{14} \mathrm{C}$-benzylamine as a substrate, and the mRNA-levels were analyzed by real-time PCR.
\end{abstract}

Results: We found that the enzyme activity was increased in lung and adipose tissue 1 day after induction, as the glucose levels start to rise. Seven days after the injection of alloxan, the activity in serum was increased, and this activity was positively correlated with blood glucose levels in the alloxan-treated animals. Although the enzyme activity was increased in adipose tissue as a result of the treatment, SSAO-mRNA levels in this tissue were decreased, possibly suggesting a negative feedback on the gene expression.

Conclusions: The main conclusion from this study is that the increased enzyme activity observed in diabetes is not a result of increased SSAO gene transcription. We speculate that the enzyme activity is controlled by posttranslational modifications of the protein, and that the catalytic activity controls the gene expression.

\section{Introduction}

Semicarbazide-sensitive amine oxidase (SSAO; EC.1.4.3.6) is a family of copper-dependent enzymes that can be found in a variety of species, both eukaryotic and prokaryotic (reviewed in Callingham et al. [1]). In mammals, SSAOs is found mainly in plasma membrane of adipocyte, smooth muscle, and endothelial cells, but also to a large extent as a soluble enzyme in plasma (reviewed in Jalkanen and Salmi [2]). For decades, SSAOs have been known as enzymes oxidizing amines in a double-displacement reaction, producing an aldehyde, hydrogen peroxide, and ammonia, but little has been known of their physiological relevance. In the last few years, however, it has become increasingly clear that SSAO has important functions as an ecto-enzyme, regulating cell signaling and adhesion, and seemingly also adipocyte differentiation (3-5). For example, the enzyme has been shown to possess adhesive properties, guiding lymphocytes to sites of inflammation (4), and it can affect glucose uptake into the cells $(6,7)$. The origin of plasma SSAO has been a matter of speculation, but data are now accumulating suggesting that the enzyme is derived, at least in part, from vascular

Address correspondence and reprint requests to: Jenny Nordquist, Department of Neuroscience, Unit of pharmacology BMC, Husargatan 3, Box 593, 75124 Uppsala, Sweden. Phone: +46 1847149 46; fax: +46 185115 40;

e-mail: Jenny.Nordquist@neuro.uu.se smooth muscle cells and endothelial cells (Göktürk et al., unpublished observations). One possible mechanism for its release into the blood stream could be proteolytic cleavage of the N-terminal, which functions as a transmembrane anchor (8).

A lot of interest has been focused on SSAO in relation to diabetes because the plasma activity of the enzyme has been observed to be about $40 \%$ higher in diabetic patients compared to healthy controls (9-12), and even higher if microvascular complications, such as retinopathy (11-13), are present. The SSAO activity appears to be increased as a result of the onset of diabetes $(14,15)$, although the underlying mechanism for this is unknown. Considering that the activity is increased long before any detectable microvascular complications arise (10), together with what is known about the properties of SSAO, many investigators have suggested that the enzyme may be directly involved in the development of vasculopathies $(16,17)$. Methylamine and aminoacetone head the list of endogenous substrates for human SSAO, yielding formaldehyde and methylglyoxal, respectively, as well as hydrogen peroxide and ammonia. These aldehydes are known to be adduct-forming and are, along with the other reaction products, considered to be cytotoxic $(16,18)$. Additionally, the lymphocyte binding capacity of SSAO is of particular interest in light of the fact that diabetic microangiopathy is associated with capillary occlusion by leukocytes adhering to vessel 
walls (19). Consequently, it has been speculated that an SSAO inhibitor could be of value in treating patients with diabetes (17).

It is not known how the SSAO activity is regulated. Previous investigations have shown that alloxan- and streptozotocin-induced diabetes cause an increase in SSAO activity $(14,15)$, but there have been no reports revealing at what level this increase is controlled. In this study, our aim was to examine if gene expression contributes to the regulation of SSAO activity. This we did by studying the activity as well as mRNA levels of SSAO in response to alloxan-induced diabetes in mice. To our surprise, however, we found that an increased enzyme activity was associated with decreased SSAO mRNA levels. To explore these new findings further, we also examined whether two SSAO inhibitors, hydralazine and carbidopa, would have an effect on the SSAO mRNA levels.

\section{Materials and Methods \\ Animals}

Male NMRI mice (outbred) weighing 30-35 g, purchased from B\&K Universal AB (Sollentuna, Sweden), were used in all experiments. The mice were housed in wire cages in a room with 12:12 hr light-dark cycles and they had free access to food (Lactamin, Solna, Sweden) and water. The temperature in the room was $21( \pm 1)^{\circ} \mathrm{C}$ and the relative humidity $50( \pm 15) \%$. All experiments were carried out with permission from the local animal ethical committee.

Diabetes was induced by a single intravenous injection of alloxan $(75 \mathrm{mg} / \mathrm{kg})$ and determined as blood glucose levels above $12 \mathrm{mmol} / \mathrm{l}$ (MediSense Pen sensor, Abbot Laboratories, Abbot Park, Illinois, USA). Two alloxan-treated animals did not reach this level and were therefore excluded from the study. The animals were sacrificed by cervical dislocation 1 or 7 days after the induction of diabetes. Tissues designated for mRNA analyses were immediately submerged in RNAlater according to the instructions from the manufacturer (Ambion, Austin, TX, USA) and subsequently frozen at $-80^{\circ} \mathrm{C}$, whereas tissues for the enzymatic analyses were frozen at $-80^{\circ} \mathrm{C}$ without any pretreatment. Blood was allowed to clot in $4^{\circ} \mathrm{C}$ for $2-4 \mathrm{hr}$ before it was centrifuged at $4^{\circ} \mathrm{C}, 2000 \mathrm{~g}$ for $5 \mathrm{~min}$. The serum supernatant was immediately analyzed for SSAO activity.

To assess the possibility of an autoregulatory mechanism, we examined if a decreased enzyme activity would affect the SSAO gene expression. Animals were therefore treated with either of two known SSAO inhibitors, hydralazine hydrochloride $(2 \times$ $0.5 \mathrm{mg}$, Sigma-Aldrich Sweden AB, Sweden), or carbidopa $(2 \times 0.5 \mathrm{mg}$, Sigma-Aldrich Sweden AB, Stockholm, Sweden). The two doses were given intraperitoneally (IP) $24 \mathrm{hr}$ apart. Three days after the last injection of hydralazine hydrochloride, carbidopa, or saline, the mice were killed by cervical dislocation. The tissues were treated as described.

\section{RNA Preparations and cDNA Synthesis}

We prepared total RNA from lung as well as from white abdominal adipose tissue by use of the kit RNeasy Mini (Qiagen, Hilden, Germany). To disrupt DNA, tissue lysates were run through QIAshredder columns (Qiagen) and were DNase treated (Qiagen) during the RNA preparations. Total RNA was eluted from the RNeasy Mini columns with $30 \mu \mathrm{l}$ of RNasefree water. The RNA-concentrations obtained were quantified fluorometrically with a Hitachi F-4000 fluorescence spectrophotometer, using the fluorescent nucleic acid stain RiboGreen (Molecular Probes, Eugene, OR, USA).

Subsequently, we synthesized cDNA using the kit Ready-To-Go You-Prime First-Strand Beads (Amersham Pharmacia Biotech, Uppsala, Sweden). For this synthesis, we used 100 ng total RNA diluted to $33.5 \mu \mathrm{l}$, and we incubated the reaction at $37^{\circ} \mathrm{C}$ for $1 \mathrm{hr}$ along with $0.66 \mu \mathrm{g}$ random hexamers (Amersham Pharmacia Biotech) and $0.5 \mu$ g oligo-dT primer (Amersham Pharmacia Biotech). After the incubation, all cDNA samples were diluted to a volume of $100 \mu \mathrm{l}$, and were stored at $-80^{\circ} \mathrm{C}$ until quantification by real-time PCR. All kits were used following the instructions from the manufacturers.

\section{Real-Time PCR}

We designed primers and a TaqMan probe for mouse SSAO (GenBank accession AF078705) using the software Primer Express (Applied Biosystems, Foster City, CA, USA) (Table 1). The primers and the FAM-labeled probe were purchased HPLC-purified from Interactiva (Interactiva Biotechnologie $\mathrm{GmbH}$, Ulm, Germany). Both primers were in exon 4 and the resulting amplicon was 69 bases long. A BLASTsearch revealed no cross-reactivity toward any other DNA-sequences in mouse.

A two-step PCR was optimized on an iCycler instrument (BioRad, Hercules, CA, USA) with 50 cycles of amplification: Denaturation at $95^{\circ} \mathrm{C}$ for 15 sec and a combined annealing and extension step at $60^{\circ} \mathrm{C}$ for $1 \mathrm{~min}$. The polymerase used was AmpliTaq Gold with PCR Buffer II (Applied Biosystems). The PCR was performed in a volume of $25 \mu \mathrm{l}$, containing $1 \mu \mathrm{l}$ template cDNA, $0.4 \mu \mathrm{M}$ of each

Table 1. Primers and probes used for real-time PCR

\begin{tabular}{llll}
\hline Gene & Primer/probe & Product \\
\hline SSAO & Forward & 5'TGCACATCCCTCAT- & \\
& & GCAGAA 3' & \\
& Reverse & 5'GGAGGAAGAAGCC- & 69 bases \\
& CACTGAGT 3' & \\
& Probe & 5'FAM-ATCCCCAACAC- & \\
& & GGTGAC & \\
& & TGCGG-TAMRA 3'
\end{tabular}


primer, $0.2 \mu \mathrm{M}$ of the FAM-labeled probe, $1.8 \mathrm{mM}$ $\mathrm{MgCl}_{2}$, and $0.32 \mathrm{mM} \mathrm{dNTP}$. When optimizing the PCR, we ran the PCR products on agarose gels and only one band, of the expected size, could be detected. No primer dimer formation was detected.

Each cDNA-sample was run on three separate PCR plates, each time in triplicate. With each run, a standard cDNA was included in quadruplicates of three different concentrations to produce a standard curve. One of the control samples from adipose tissue was used to produce this standard CDNA, because this tissue, by far, has the highest expression of SSAO. Additionally, for every 96-well reaction plate, negative controls without template were added.

The samples were screened for contaminating genomic DNA by using the RNA preparations as templates in the real-time PCR. We found that most samples had no detectable genomic DNA, but in some, amplification was observed. However, the highest concentrations of contaminating DNA were still about 80 times lower than the amount of cDNA after cDNA synthesis, and we therefore concluded that these very low levels could be disregarded.

\section{Analysis of Data from Real-Time PCR}

The threshold cycle $\left(C_{\mathrm{T}}\right)$ data obtained from running real-time PCR was related to a standard curve in order to obtain the starting quantity (SQ) of the template cDNA in each sample. Standard curves were established by plotting $C_{\mathrm{T}}$ values against the $\log 10$ of the total RNA concentration in the standard cDNA. The slope values of the standard-curve ranged from -3.2 to -3.6 .

Because $28 \mathrm{~S}$ ribosomal RNA as well as a number of housekeeping genes appeared to be affected by the diabetes induction as well as by the treatment with SSAO inhibitors in our sample (data not shown), we decided not to use any of these for normalizing our data. Instead, we used total RNA, quantified fluorometrically, using RiboGreen (Molecular Probes) as the internal standard. However, this internal standard does not correct for variations in the efficiency of the cDNA synthesis. We therefore took measures to avoid inaccurate results due to variations in this step. First of all, cDNA was synthesized from one tissue in one set of animals all at once, so that all samples to be compared were synthesized simultaneously. Second, this step was repeated once for all samples. Differences in SSAO mRNA between diabetic or inhibitor-treated animals and controls were considered statistically significant only if the $p$-values obtained from both cDNA sets were $<0.05$.

\section{Analysis of SSAO Activity}

Preparing for the SSAO activity analyses, the tissues were thawed, submerged in $0.1 \mathrm{M}$ Na-phosphate buffer, and homogenized with a Dounce homogeniser. The $5 \%$ homogenates obtained were frozen at $-80^{\circ} \mathrm{C}$ until the time of analysis.
We measured SSAO activity both in serum and in tissue homogenates according to a method described by McCaman et al. using ${ }^{14} \mathrm{C}$-benzylamine as a substrate (20). Serum samples were analyzed without any pretreatment, whereas the tissue homogenates were thawed and sonicated with a Branson Sonifier Cell disrupter Bl before analysis. Additionally, the protein content of the tissue samples was determined, using the Lowry method (21) as a means of normalizing the values. Briefly, $50 \mu \mathrm{l}$ of $5 \%$ homogenate was incubated at room temperature with $25 \mu \mathrm{l} 0.2 \mathrm{mM}$ clorgyline and $25 \mu \mathrm{l}$ $0.1 \mathrm{mM}$ deprenyl. The reaction was started when 50 $\mu \mathrm{l}{ }^{14} \mathrm{C}$-benzylamine was added. The samples were incubated in $37^{\circ} \mathrm{C}$ for $20 \mathrm{~min}$ before the reaction was terminated by the addition of $30 \mu \mathrm{l} \mathrm{M} \mathrm{HCl}$. The enzyme activity in serum is given as nmol benzylamine oxidized $\cdot \mathrm{ml}^{-1} \cdot \mathrm{h}^{-1}$ and as nmol benzylamine oxidized.mg protein ${ }^{-1} \cdot h^{-1}$ in all tissue homogenates except for adipose tissue. The activity in adipose tissue is expressed as nmol benzylamine oxidized.mg tissue $^{-1} \cdot \mathrm{h}^{-1}$ because protein content could not be quantified accurately in this particular tissue.

\section{Statistics}

Groups were compared using simple linear regression or Student's unpaired $t$-test. All statistical analyses were performed using the MacIntosh software Statview 4.5 (SAS Institute, Inc., Cary, NC, USA).

\section{Results}

Of the tissues examined, adipose tissue displayed the highest basal SSAO activity. Compared to lung tissue, the activity was approximately six times higher in adipose tissue (Table 2 ).

As early as $24 \mathrm{hr}$ after the induction of diabetes, as the blood glucose levels were rising, SSAO activity was increased in adipose and lung tissue by $66 \%$ and $34 \%$, respectively. At this time point, however, the activity in serum was unaffected. Seven days after the induction of diabetes, the activity in adipose tissue was still higher (42\% higher than controls), the activity in serum was increased by $24 \%$, whereas the levels had

Table 2. Comparison of enzyme activity and mRNA levels in lung and adipose tissue

\begin{tabular}{lccc}
\hline & Adipose & Lung & $\begin{array}{c}\text { Adipose/ } \\
\text { lung }\end{array}$ \\
\hline SSAO activity & $1.78(0.46)$ & $0.29(0.13)$ & 6 \\
SSAO mRNA & $1.81(0.78)$ & $0.013(0.009)$ & 139
\end{tabular}

Mean (SD) of all controls. Values for mRNA represent starting quantity. SSAO activity is given as nmol.mg tissue ${ }^{-1} \cdot \mathrm{h}^{-1}$ 
a) Lung tissue

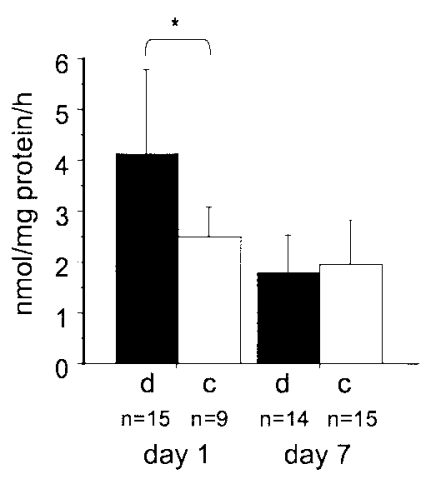

b) Adipose tissue

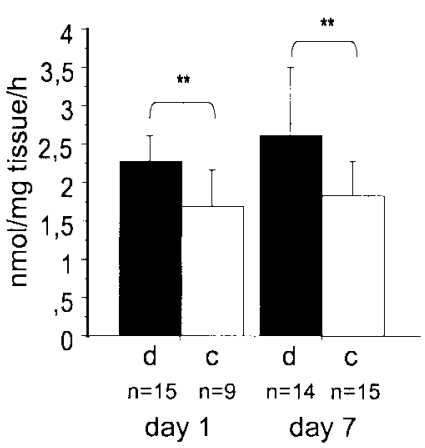

c) Serum

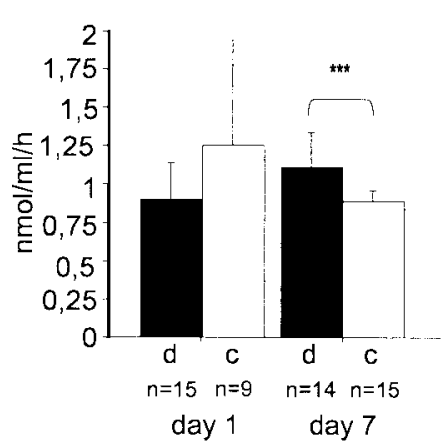

Fig. 1. SSAO activity in lung and adipose tissue, and serum 1 and 7 days after induction of diabetes. Please note that the unit on the $y$-axes differs in (a), (b) and (c) and the graphs can therefore not be compared directly. Abbreviations: d, alloxan-induced diabetes; c, controls. ${ }^{*} p<0.05,{ }^{* *} p<0.01,{ }^{* * *} p<0.001$. Error bars represent the upper half of the standard deviations.

returned to normal in lung tissue (Fig. 1). In addition to these tissues, we examined the SSAO activity in pancreas and small intestine, but neither of these two tissues showed any altered activity as a result of the alloxan treatment (data not shown).

Linear regression suggests that the SSAO activity in serum is positively correlated with blood glucose levels $(p=0.0499) 7$ days after the induction of diabetes (Fig. 2). We performed linear regression on data from the alloxan-treated animals only.

SSAO mRNA was quantified in two sets of cDNA (prepared twice from the same RNA) by realtime PCR. These analyses showed that the mRNA levels were approximately 140 times higher in adipose compared to lung tissue (Table 2). We also found that the mRNA levels were lower in adipose tissue from diabetic animals compared to controls as early as 1 day after the alloxan injection $(p=0.0101$ and 0.0017, for cDNA set 1 and 2, respectively),

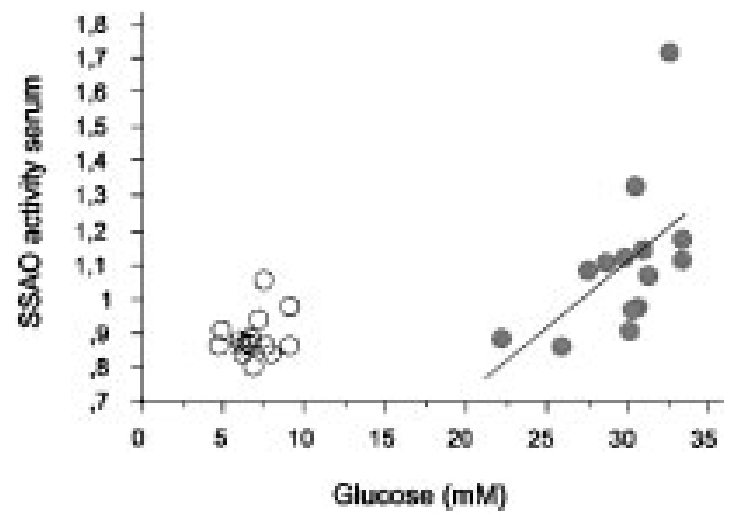

Fig. 2. SSAO activity in serum $\left(\mathrm{nmol} \cdot \mathrm{ml}^{-1} \cdot \mathrm{h}^{-1}\right) \mathbf{7}$ days after the induction of diabetes plotted against blood glucose levels at the same time point. The alloxan-treated animals (filled circles, $n=14$ ) are separated from the controls (open circles, $n=15$ ), with blood glucose levels exceeding $12 \mathrm{mM}$. Linear regression on the group of diabetic animals (regression line in the plot) showed a positive correlation between SSAO activity and glucose concentrations $\left(p<0.05, r^{2}=0.284\right)$. and that this effect was maintained 7 days after the injection ( $p=0.0105$ and 0.0015) (Fig. 3). In lung tissue, the mean mRNA levels were lower 1 day after the induction of diabetes, although this was not statistically significant $(p=0.07$ and 0.25$)$. After 7 days with diabetes, the mRNA levels in lung were higher in the diabetic animals $(p=0.0385$ and 0.0308). Summarizing these findings, the tendency is that increased SSAO activity is associated with lower SSAO mRNA levels in adipose tissue. The mRNA levels in lung tissue, however, did not display any signs of being positively or negatively correlated with the enzyme activity.

Treating animals with the SSAO-inhibitors hydralazine hydrochloride or carbidopa, did not result in significantly altered levels of SSAO mRNA (Fig. 4).

\section{Discussion}

This study has provided evidence that the increased enzyme activity observed in alloxan-induced diabetes cannot be ascribed to increased gene transcription. Nevertheless, it may well be that there is some degree of genetic regulation involved; our data indicate that increased enzyme activity is associated with a downregulation of SSAO mRNA in adipose tissue. A plausible explanation for this finding could be that the gene transcription is under the control of an autoregulatory feedback loop, perhaps mediated by the reaction product hydrogen peroxide, which is known to affect transcription factors such as NF $\kappa \mathrm{B}$ (reviewed in Bauerle and Henkel [22]). However, because we were unable to provoke higher mRNA levels in adipose tissue by treating animals with SSAO inhibitors, we cannot prove the existence of such a product-mediated feedback loop. One explanation for this result is that the negative feedback is not related to the products in the enzyme reaction, but rather to the amount of catalytically active protein. Another possibility is that the negative feedback is sensitive only to increased enzyme activity as a way of preventing overproduction of enzyme or reaction products. Thus, our data imply 
a) Lung tissue, day 1

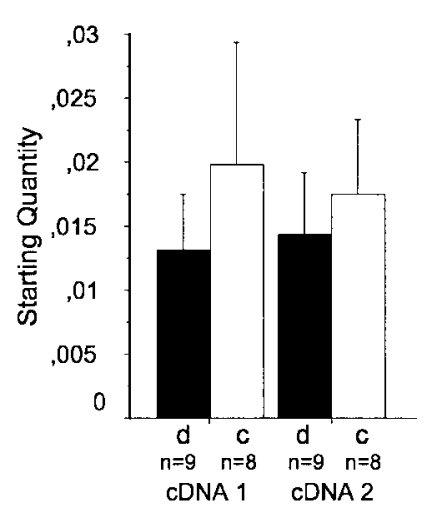

c) Adipose tissue, day 1

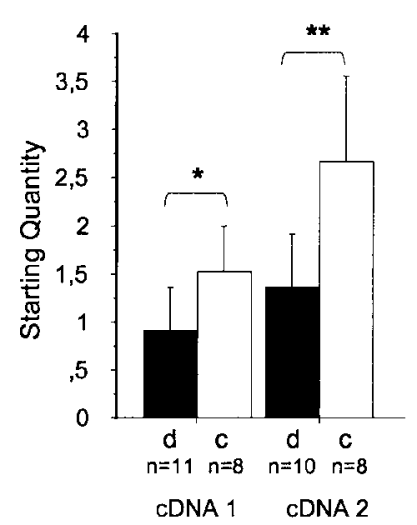

b) Lung tissue, day 7

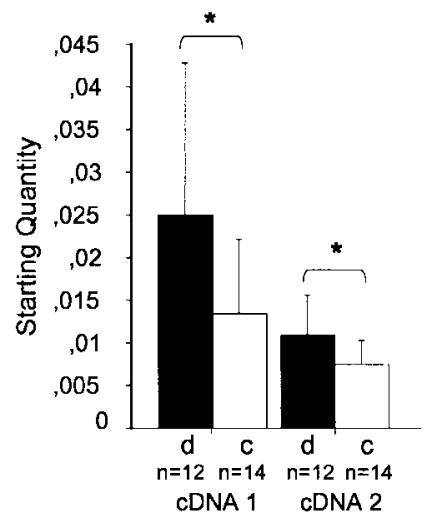

d) Adipose tissue, day 7

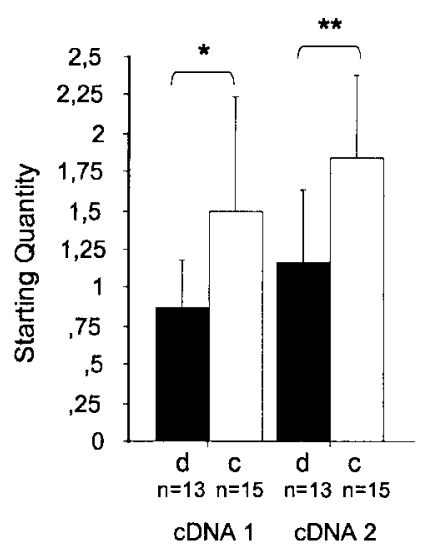

Fig. 3. SSAO-mRNA (SQ, starting quantity) in lung- and adipose tissue, one and seven days after induction of diabetes. Two cDNA-preparations were used. The differences between controls and alloxan-treated animals were considered statistically significant only when $p<0.05$ in both sets of cDNA. d: alloxan-induced diabetes; c: controls. ${ }^{*} p<0.05,{ }^{* *} p<0.01$. Error bars represent the upper half of the standard deviations.

that there is a self-regulatory mechanism, but we can only speculate regarding the identity of the mediators controlling it.

We did not find support for a negative feedback loop in lung tissue. Considering that the enzyme activity, as well as the mRNA levels, was much lower in this tissue, a negative feedback loop would be more difficult to detect and would perhaps have become evident only with a larger sample. However, we cannot exclude the possibility that SSAO gene transcription is, in fact, controlled differently in lung. Previous investigations have shown that the recovery rate for SSAO activity differs extensively between different tissues after irreversible inhibition of the enzyme, presumably reflecting different rates of de novo synthesis of the protein (23).

We have presented data suggesting that the increased SSAO activity seen in diabetes is regulated posttranscriptionally, and consequently, posttransla-

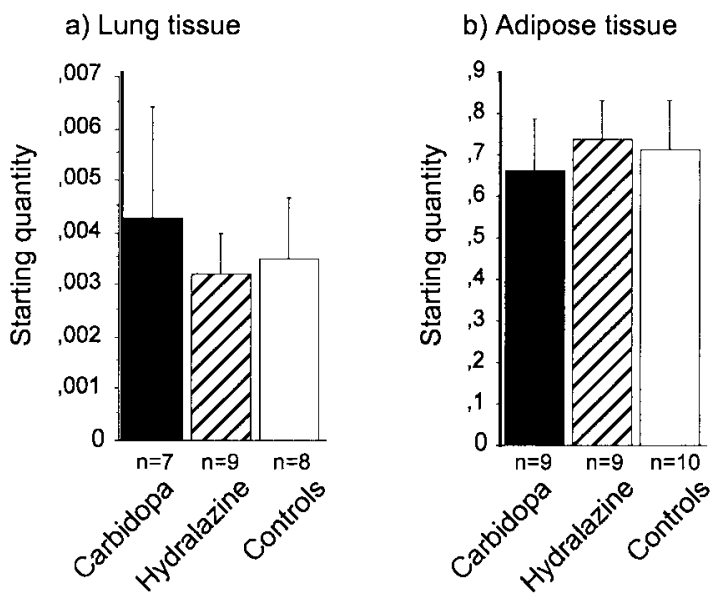

Fig. 4. SSAO-mRNA (SQ, starting quantity) in lung- and adipose tissue after treatment with saline (controls), hydralazine hydrochloride $(2 \times 0.5 \mathrm{mg})$ or carbidopa $(2 \times 0.5 \mathrm{mg})$. Error bars represent the upper half of the standard deviations.

tional modifications of the protein must be considered as a potential site for regulation. It is known, from previous studies, that $V_{\max }$ is increased in diabetes, whereas the $K_{\mathrm{m}}$ is essentially unchanged (14). In other words, it is the maximum capacity of the enzyme pool that is increased, probably by an increased number of catalytically active SSAO molecules. Changes in the glycosylation pattern would result in a changed tertiary structure of the protein, which in turn, could stabilize it in an active state or impair its proteolysis. The cofactors, copper and TOPA-quinone, are other important candidates when posttranslational modifications are considered. In our sample, the SSAO activity in serum is increased only after the activity in lung and adipose tissue is increased. This may suggest that activation of the enzyme takes place in the tissues before it is released into the bloodstream. Furthermore, we found that the SSAO activity in serum is positively correlated with blood glucose levels, implying that glucose may play a role either in activating the enzyme or in regulating its release from the blood vessel wall. The activation of SSAO in diabetes is of particular interest with regard to its involvement in glucose transport, and consequently, a pertinent question is whether this increased enzyme activity leads to a subsequent increase in glucose uptake into the cells.

Another finding worth noting in the present study is that the SSAO mRNA levels in adipose tissue is more than 100-fold higher than the mRNA levels in lung tissue, whereas the enzyme activity in adipose tissue is only six times higher. There are a number of possible explanations for this, and we can only speculate as to which is the most conceivable. It may be that there is a large pool of inactive enzyme in adipose tissue, that SSAO produced here is exported to other tissues, that the protein is degraded more rapidly, or that the degree of translation 
is lower. The proportion of the increased activity is higher in lung tissue 1 day after the alloxan injection as compared to adipose tissue, but this increase is not sustained. One explanation may be that the direct response to diabetes is higher in lung, whereas the pool potential in this tissue is low. The response in adipose tissue is more moderate, perhaps due to the high baseline activity, and more durable, perhaps due to a large pool of enzyme. This may be an appealing thought, but we cannot rule out the possibilities of a lower degree of translation or a faster degradation. We amplified $28 \mathrm{~S}$ rRNA in adipose as well as in lung tissue and it appears that there is about six times more $28 \mathrm{~S}$ rRNA in lung compared to adipose tissue (data not shown). This could be indicative of fewer ribosomes in this particular tissue.

The SSAO molecule is large, a $220-\mathrm{kDa}$ homodimer (24), and is produced in considerable amounts, especially in adipose tissue. Estimations have been made suggesting that there are about 14 million SSAO molecules on the surface of one single adipocyte, and that this constitutes $2.3 \%$ of the total protein content in the plasma membrane of these cells (25). Synthesizing such a large molecule, in such large quantities must cost a lot for the cell, and is undoubtedly of great importance. It would be expected that no more than what is needed is produced, and consequently, the regulation of its synthesis must be of great importance. Here, we have reported the possibility that the gene expression is regulated by the enzyme it is encoding.

\section{Acknowledgments}

We wish to thank Astrid Nordin for technical assistance and Dr Sofia Mikko for invaluable discussions regarding real-time PCR. This study was supported with grants from The Swedish Medical Research Council (no. 4145).

\section{References}

1. Callingham BA, Crosbie AE, Rous BA. (1995) Some aspects of the pathophysiology of semicarbazide-sensitive amine oxidase enzymes. Prog. Brain Res. 106: 305-321.

2. Jalkanen S, Salmi M. (2001) Cell surface monoamine oxidases: enzymes in search of a function. Embo. J. 20: 3893-3901.

3. Enrique-Tarancon G, Marti L, Morin N, et al. (1998) Role of semicarbazide-sensitive amine oxidase on glucose transport and GLUT4 recruitment to the cell surface in adipose cells. J. Biol. Chem. 273: 8025-8032.

4. Salmi M, Tohka S, Berg EL, et al. (1997) Vascular adhesion protein 1 (VAP-1) mediates lymphocyte subtype-specific, selectinindependent recognition of vascular endothelium in human lymph nodes. J. Exp. Med. 186: 589-600.

5. Moldes M, Feve B, Pairault J. (1999) Molecular cloning of a major mRNA species in murine 3T3 adipocyte lineage. Differentiation-dependent expression, regulation, and identification as semicarbazide-sensitive amine oxidase. J. Biol. Chem. 274: 9515-9523.

6. Marti L, Morin N, Enrique-Tarancon G, et al. (1998) Tyramine and vanadate synergistically stimulate glucose transport in rat adipocytes by amine oxidase-dependent generation of hydrogen peroxide. J. Pharmacol. Exp. Ther. 285: 342-349.
7. El Hadri K, Moldes M, Mercier N, et al. (2002) Semicarbazidesensitive amine oxidase in vascular smooth muscle cells: differentiation-dependent expression and role in glucose uptake. $A r-$ terioscler. Thromb. Vasc. Biol. 22: 89-94.

8. Salminen TA, Smith DJ, Jalkanen S, Johnson MS. (1998) Structural model of the catalytic domain of an enzyme with cell adhesion activity: human vascular adhesion protein-1 (HVAP1) D4 domain is an amine oxidase. Protein Eng. 11: 1195-1204.

9. Tryding N, Nilsson SE, Tufvesson G, et al. (1969) Physiological and pathological influences on serum monoamine oxidase level. Effect of age, sex, contraceptive steroids and diabetes mellitus. Scand. J. Clin. Lab. Invest. 23: 79-84.

10. Boomsma F, van den Meiracker AH, Winkel S, et al. (1999) Circulating semicarbazide-sensitive amine oxidase is raised both in type I (insulin-dependent), in type II (non-insulindependent) diabetes mellitus and even in childhood type I diabetes at first clinical diagnosis. Diabetologia 42: 233-237.

11. Garpenstrand H, Ekblom J, Bäcklund LB, et al. (1999) Elevated plasma semicarbazide-sensitive amine oxidase (SSAO) activity in Type 2 diabetes mellitus complicated by retinopathy. Diabet Med. 16: 514-521.

12. Boomsma F, Derkx FH, van den Meiracker AH, et al. (1995) Plasma semicarbazide-sensitive amine oxidase activity is elevated in diabetes mellitus and correlates with glycosylated haemoglobin. Clin. Sci. 88: 675-679.

13. Grönvall-Nordquist JL, Bäcklund LB, Garpenstrand H, et al. (2001) Follow-up of plasma semicarbazide-sensitive amine oxidase activity and retinopathy in Type 2 diabetes mellitus. J. Diabetes Complications 15: 250-256.

14. Hayes BE, Clarke DE. (1990) Semicarbazide-sensitive amine oxidase activity in streptozotocin diabetic rats. Res. Commun. Chem. Pathol. Pharmacol. 69: 71-83.

15. Elliott J, Fowden AL, Callingham BA, et al. (1991) Physiological and pathological influences on sheep blood plasma amine oxidase: effect of pregnancy and experimental alloxan-induced diabetes mellitus. Res. Vet. Sci. 50: 334-339.

16. Yu PH, Zuo DM. (1993) Oxidative deamination of methylamine by semicarbazide-sensitive amine oxidase leads to cytotoxic damage in endothelial cells. Possible consequences for diabetes. Diabetes 42: 594-603.

17. Ekblom J. (1998) Potential therapeutic value of drugs inhibiting semicarbazide-sensitive amine oxidase: vascular cytoprotection in diabetes mellitus. Pharmacol. Res. 37: 87-92.

18. Thornalley PJ, Westwood M, Lo TW, McLellan AC. (1995) Formation of methylglyoxal-modified proteins in vitro and in vivo and their involvement in AGE-related processes. Contrib. Nephrol. 112: 24-31.

19. Schröder S, Palinski W, Schmid-Schönbein GW. (1991) Activated monocytes and granulocytes, capillary nonperfusion, and neovascularization in diabetic retinopathy. Am. J. Pathol. 139: $81-100$.

20. McCaman RE, McCaman MW, Hunt JM, Smith MS. (1965) Microdetermination of monoamine oxidase and 5-hydroxytryptophan decarboxylase activities in nervous tissues. J. Neurochem. 12: 15-23.

21. Lowry O, Rosebrough N, Farr A, Randall R. (1951) Protein measurement with the Folin phenol reagent. J. Biol. Chem. 193: 265-275.

22. Baeuerle PA, Henkel T. (1994) Function and activation of NFkappa B in the immune system. Annu. Rev. Immunol. 12: 141-179.

23. Grönvall JL, Garpenstrand H, Oreland L, Ekblom J. (1998) Autoradiographic imaging of formaldehyde adducts in mice: possible relevance for vascular damage in diabetes. Life Sci. 63: 759-768.

24. Bono P, Jalkanen S, Salmi M. (1999) Mouse vascular adhesion protein 1 is a sialoglycoprotein with enzymatic activity and is induced in diabetic insulitis. Am. J. Pathol. 155: 1613-1624.

25. Morris NJ, Ducret A, Aebersold R, et al. (1997) Membrane amine oxidase cloning and identification as a major protein in the adipocyte plasma membrane. J. Biol. Chem. 272: 93889392. 